previously normotensive mother (systolic blood pressure of $\geq 140 \mathrm{~mm} \mathrm{Hg}$, diastolic of $\geq 90 \mathrm{~mm} \mathrm{Hg}$ ). Data were collected from maternal healthcare centers to antenatal clinic records. Assessment of the child's intellectual level was based on psychometric test results collected from multiple sources.

Results In multivariate analyses, maternal GH (OR 2.37, 95\% CI 1.44 to 3.89 ) and pre-pregnancy obesity (OR 2.20, 95\% CI 1.24 to 3.89) were independent risk factors associated with MCL in addition to high parity, familial socio-economic status lower than professional and small birth weight for gestational age of the newborn.

Conclusions Besides factors indicating adverse maternal health and socio-economic status during pregnancy, also maternal GH should be considered as one of the early risk factors, which may predispose to impaired cognitive development in childhood. However, replication study is needed for delineating the association between maternal GH and MCL in the offspring.

\section{P1-443 REPRODUCTIVE TRACT INFECTIONS (RTI) AMONG LOW SOCIOECONOMIC COMMUNITY WOMEN IN COLOMBO, SRI LANKA}

doi:10.1136/jech.2011.142976g.33

${ }^{1} S$ Herath, ${ }^{2}$ P Fonseka, ' $S$ Samarakoon. 'National STD/AIDS Control Programme, Colombo, Sri Lanka; ${ }^{2}$ Dept of Community Medicine, Medical Faculty, University of Sri Jayawardenapura, Colombo, Sri Lanka

Introduction Community prevalence data on RTI are sparse and it is believed to be high among low socio economic communities. This study was carried out to describe the epidemiological characteristics of selected RTI among the reproductive age women living in low socioeconomic communities within the Colombo Municipal Council (CMC).

Methodology A representative sample of seven hundred and seventy women was selected using a multistage sampling procedure from fifteen randomly selected Public Health Midwives (PHMM) areas from five Municipal Districts. An interviewer administered questionnaire was used to obtain data on socicodemographic factors, economic factors, and behavioural correlates. Clinical examination of women was carried out at the Primary HealthCare clinics (PHC) of the CMC. Biological specimens were collected for laboratory diagnosis. Results Ninety-six per cent (96\%) of women participated in the study. Endogenous infections and candidiasis were relatively common being (BV 8.6\%, 95\% CI 6.6 to 10.6 , and $6.8 \% 95 \%$ CI 5 to 8.6) respectively. Sexually Transmitted Infections (STI) were infrequent. GC $-0 \%$, CT $0.6 \%$ (95\% CI 0.04 to 1.16$)$, and TV $0.6 \%(95 \%$ CI 0.04 to 1.16$)$. Of the symptom related correlates investigated, women's complaints of itching of vulva ( $p=0.014, \mathrm{OR}=3.198,95 \%$ CI 1.260 to 8.114 ) and vaginal discharge ( $p=0.026, O R=2.611,95 \%$ CI 1.121 to 6.083 ) were statistically significantly associated with candidiasis in univariate analysis. However, this finding was not statistically significant in the multivariate analysis.

Conclusion Unlike the common believe, this community based study demonstrated a low prevalence of RTI, among the urban slum dwellers in CMC.

\section{P1-444 LIVING ARRANGEMENTS ARE A FACTOR OF JAPANESE LONGEVITY? AGES COHORT STUDY}

doi:10.1136/jech.2011.142976g.34

${ }^{1} \mathrm{H}$ Hirai, ${ }^{1} \mathrm{~K}$ Kondo, ${ }^{2} \mathrm{~T}$ Ojima, ${ }^{3} \mathrm{~A}$ Hata. ${ }^{1}$ Nihon Fukushi University, Nagoya, Japan; ${ }^{2}$ Hamamastu University school of medicine, Hamamamtsu, Japan; ${ }^{3}$ Chiba University, Chiba, Japan

Introduction The average life expectancy of the Japanese is the highest in the world. What makes Japan different from other countries? According to International Comparison Survey in 2005, one of characteristics of Japanese elderly was low proportion of those who live alone $(11.0 \%$ in Japan, $35.4 \%$ in USA, $38.6 \%$ in France). This study aims to examine relationship between living arrangement and healthy life expectancy among the elderly in Japan. Methods This study is based on data from the Aichi Gerontological Evaluation Study(AGES) Project. We analysed 13295 persons aged 65 or older who were functionally independent at baseline. The endpoint was loss of health (death or functional decline) during follow-up period of 48 months. Independent variables were living arrangements, age, years of current address, income, diseases, body mass index, smoking, drinking, walking, and depression. Cox regression was used to calculate the HR. Analyses were stratified by gender. Result Cox regression showed significant higher HR of loss of health for living alone ( $\mathrm{HR}=1.54$ for men, $\mathrm{HR}=1.41$ for women) compared to those who living with spouse and children after controlling for age. After controlling for other variables, significant HR were shown for living alone ( $\mathrm{HR}=1.32$ for men, $\mathrm{HR}=1.31$ for women).

Conclusions Living arrangements were significantly rerated to loss of health. The norm of parent-child co-residence possibly has contributed to Japanese longevity up to the present. These results suggest that the recent increasing trend in number of the elderly living alone can result ruin health of Japanese in the future.

\section{P1-445 CONNECTING SPACES: THE FUNCTION OF PLACE FOR NEIGHBOURHOOD PHYSICAL ACTIVITY}

doi:10.1136/jech.2011.142976g.35

V Ivory, C Woodham, T Blakely.* University of Otago, Wellington, New Zealand

Introduction This study examined the relationship between neighbourhoods and physical activity in New Zealand. We investigated the role of local public places in the lives of residents and how they impact on physical activity.

Methods Four case study neighbourhoods were selected varying by deprivation and the level of physical activity resources. Key informant interviews with 21 local government and health promotion practitioners and fourteen focus groups were conducted across the four neighbourhoods with adult residents, varying by exposure to the neighbourhood, gender and ethnicity (Māori and non-Māori).

Results A consistent theme across neighbourhoods was that local destinations and public places were seen as opportunities to be social-to connect, interact and meet people. While not everyone wanted to be active with or around others, many of the participants that enjoyed being active within their neighbourhood were more motivated to do so as part of group, or as family/whanāu, and valued being active in places where other people were present, as opposed to being alone. Having quality, multi-use, public places nearby made it easier for residents to physically active.

Conclusion The results of this study highlight the perceived importance of social connection and social interaction for physical activity levels, rather than health reasons. Whether people use public places for physical activity may be dependent on how well those places allow for interaction and collective activity. These findings are important for urban planners, local government and health practitioners who wish to design spaces that facilitate and promote physical activity.

\section{P1-446 COMPARISON OF POSTMENOPAUSAL ENDOGENOUS SEX HORMONES AMONG JAPANESE, JAPANESE BRAZILIANS, AND NON-JAPANESE BRAZILIANS}

doi:10.1136/jech.2011.142976g.36

${ }^{1} \mathrm{M}$ Iwasaki, ${ }^{2} \mathrm{Y}$ Kasuga, ${ }^{3} \mathrm{G}$ Hamada, ${ }^{1} \mathrm{~S}$ Tsugane. ${ }^{1}$ Epidemiology and Prevention Division, Research Center for Cancer Prevention and Screening, National Cancer 